\title{
Substituting a copper atom modifies the melting of aluminum clusters
}

\author{
Baopeng Cao, ${ }^{1}$ Anne K. Starace, ${ }^{1}$ Colleen M. Neal, ${ }^{1}$ Martin F. Jarrold, ${ }^{1, a)}$ Sara Núñez, ${ }^{2}$ \\ José M. López, ${ }^{2}$ and Andrés Aguado ${ }^{2, b)}$ \\ ${ }^{1}$ Chemistry Department, Indiana University, 800 E. Kirkwood Ave., Bloomington, Indiana 47405, USA \\ ${ }^{2}$ Departamento de Física Teórica, Universidad de Valladolid, Valladolid 47011, Spain
}

(Received 1 July 2008; accepted 12 August 2008; published online 25 September 2008)

\begin{abstract}
Heat capacities have been measured for $\mathrm{Al}_{n-1} \mathrm{Cu}^{-}$clusters $(n=49-62)$ and compared with results for pure $\mathrm{Al}_{n}^{+}$clusters. $\mathrm{Al}_{n-1} \mathrm{Cu}^{-}$and $\mathrm{Al}_{n}^{+}$have the same number of atoms and the same number of valence electrons (excluding the copper d electrons). Both clusters show peaks in their heat capacities that can be attributed to melting transitions; however, substitution of an aluminum atom by a copper atom causes significant changes in the melting behavior. The sharp drop in the melting temperature that occurs between $n=55$ and 56 for pure aluminum clusters does not occur for the $\mathrm{Al}_{n-1} \mathrm{Cu}^{-}$analogs. First-principles density-functional theory has been used to locate the global minimum energy structures of the doped clusters. The results show that the copper atom substitutes for an interior aluminum atom, preferably one with a local face-centered-cubic environment. Substitution does not substantially change the electronic or geometric structures of the host cluster unless there are several $\mathrm{Al}_{n}^{+}$isomers close to the ground state. The main structural effect is a contraction of the bond lengths around the copper impurity, which induces both a contraction of the whole cluster and a stress redistribution between the $\mathrm{Al}-\mathrm{Al}$ bonds. The size dependence of the substitution energy is correlated with the change in the latent heat of melting on substitution.
\end{abstract}

(C) 2008 American Institute of Physics. [DOI: 10.1063/1.2977874]

\section{INTRODUCTION}

The melting of nanoclusters has received considerable attention in the last few years. ${ }^{1-22}$ Nanoclusters behave differently from larger particles. For example, the well-known melting point depression that occurs with decreasing particle size $^{23}$ breaks down for clusters with less than several hundred atoms. ${ }^{24-29}$ Alloy clusters can have structures and properties that are different from their pure counterparts and there has been a lot of interest in their melting behavior as well. ${ }^{30-43}$ However, almost all previous work on the melting of alloy clusters has been computational. There have been many interesting predictions. For example, Mottet et al. found that substituting a single nickel or copper impurity into icosahedral silver clusters causes a substantial increase in the melting temperature which can be attributed to the strain relaxation induced by sequestering the small impurity atom in the center of the icosahedron. ${ }^{33}$ Cheng et al. found a similar result for the substitution of a single copper atom in icosahedral $\mathrm{Au}_{55}{ }^{30}$ They also found that the copper segregates away from the surface in the liquidlike forms of $\mathrm{Au}_{54} \mathrm{Cu}$ and $\mathrm{Au}_{43} \mathrm{Cu}_{12}$.

The only previous experimental study of alloy cluster melting was reported by Neal et al. who compared the melting behavior of $\mathrm{Ga}_{n-1} \mathrm{Al}^{+}$clusters with that of pure $\mathrm{Ga}_{n}^{+}$ clusters. ${ }^{44}$ In this case, however, the melting temperatures and latent heats are not significantly influenced by the substitution. This result was rationalized by noting that gallium

\footnotetext{
${ }^{a)}$ Electronic mail: mfj@indiana.edu.

${ }^{b)}$ Electronic mail: aguado@metodos.fam.cie.uva.es.
}

and aluminum are in the same group in the periodic table, and the atoms have similar sizes (when one considers the densities of the liquids).

Here, we report studies of aluminum clusters where we substitute a copper atom for an aluminum atom. To retain the same number of valence electrons in the cluster and hence avoid possible electronic effects, we compare $\mathrm{Al}_{n-1} \mathrm{Cu}^{-}$anions and $\mathrm{Al}_{n}^{+}$cations. The copper atom is around $10 \%$ smaller than an aluminum atom and so it is possible that the copper atom in $\mathrm{Al}_{54} \mathrm{Cu}^{-}$could be sequestered in the center and stabilize an icosahedron. To complement the experimental studies, calculations were performed at the Kohn-Sham densityfunctional-theory level to search for the global minimum energy structures for $\mathrm{Al}_{n-1} \mathrm{Cu}^{-}$clusters with $n=49-62$. These results are compared to recent calculations for the aluminum cluster cations. ${ }^{45}$

\section{EXPERIMENTAL METHODS}

The heat capacities for $\mathrm{Al}_{n-1} \mathrm{Cu}^{-}$clusters were measured using multicollision-induced dissociation. The method and apparatus have been described in detail elsewhere..$^{29,44,47}$ Cluster anions are generated by laser vaporization source of a liquid alloy target. ${ }^{48}$ The aluminum/copper alloy was prepared by heating an aluminum rod and copper shot to above the melting point of the alloy in a $0.625 \mathrm{~cm}$ diameter ceramic tube in a diffusion pumped vacuum line for more than $24 \mathrm{~h}$. After cooling, the samples were removed from the ceramic tube and a short length was cut off with a hack saw and installed into the source sample holder. The alloy used for 
the work reported here has a $\mathrm{Cu} / \mathrm{Al}$ ratio of around 4 at. \%. With this composition, aluminum clusters with one copper atom are the most abundant alloy clusters.

After formation, the alloy cluster ions are carried through the source by the helium buffer gas flow and into the temperature variable extension where their temperature is set for the heat capacity measurements. Cluster ions that exit the extension are focused into a quadrupole mass spectrometer where a particular cluster size is selected. The size-selected clusters are then focused into a high pressure collision cell where collisions with the helium cause the clusters to dissociate, if their initial translational energy is large enough. Fragment ions and intact cluster ions are swept across the collision cell by a weak electric field and those that exit are analyzed in a second quadrupole mass spectrometer. The $\mathrm{Al}_{n-1} \mathrm{Cu}^{-}$clusters dissociate by sequential loss of aluminum atoms - the cluster fragment retains the copper atom,

$$
\begin{aligned}
& \mathrm{Al}_{n-1} \mathrm{Cu}^{-} \rightarrow \mathrm{Al}_{n-2} \mathrm{Cu}^{-}+\mathrm{Al}, \\
& \mathrm{Al}_{n-2} \mathrm{Cu}^{-} \rightarrow \mathrm{Al}_{n-3} \mathrm{Cu}^{-}+\mathrm{Al},
\end{aligned}
$$

etc.

The negative charge remains on the cluster fragment because it has a larger electron affinity than the isolated atom.

The fraction of the cluster ions that dissociate is determined from the mass spectrum. The fraction is measured as a function of the cluster ion's translational energy at the entrance of the collisions cell. The translational energy required for $50 \%$ of the cluster ions to dissociate (TE50\%D) is then obtained from a linear regression.

TE50\%D is measured as a function of the cluster's initial temperature (as set in the temperature variable extension). As the temperature is raised, it decreases due to the increase in the cluster's initial internal energy. A sharp decrease in TE50\%D occurs at the melting temperature due to the latent heat. The derivative of TE $50 \% \mathrm{D}$ with respect to temperature is proportional to the heat capacity. The proportionality constant is related to the fraction of the ion's translational energy that is converted into internal energy in collisions with the helium collision gas, which is estimated from a modified impulsive collision model. ${ }^{49-51}$

\section{EXPERIMENTAL RESULTS}

Figure 1 shows plots of the heat capacities measured for $\mathrm{Al}_{n-1} \mathrm{Cu}^{-}$clusters as a function of temperature. The heat capacities are plotted relative to the classical value $3 N k_{B}$, where $3 N=3 n-6+3 / 2, n$ is the total number of atoms in the cluster and $k_{B}$ is the Boltzmann constant. The filled red squares show the measured values for $\mathrm{Al}_{n-1} \mathrm{Cu}^{-}$clusters while the solid red lines show spline fits. For comparison, the open black squares in the figure show heat capacities recorded for $\mathrm{Al}_{n}^{+}$clusters. ${ }^{46,52}$ The thin solid black lines show spline fits. The plots are labeled with the total number of atoms in the cluster (n). The dashed lines in Fig. 1 show heat capacities derived from a modified Debye model that takes into account the finite size of the cluster. ${ }^{53}$

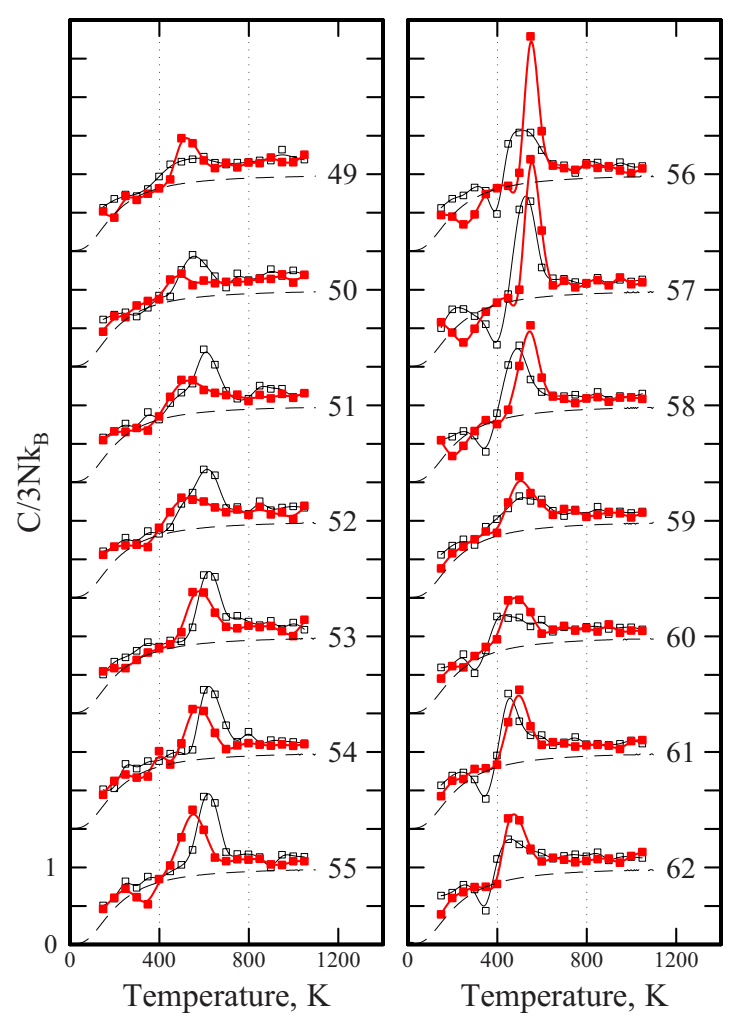

FIG. 1. (Color online) Plots of the heat capacities determined for $\mathrm{Al}_{n-1} \mathrm{Cu}^{-}$ clusters as a function of temperature. The heat capacities are plotted in terms of the classical value $3 N k_{B}$, where $3 N=3 n-6+3 / 2, n$ is the total number of atoms in the cluster, and $k_{B}$ is the Boltzmann constant. The spacing between the tick marks on the vertical axes is $3 N k_{B} / 2$. The filled red squares show the measured values for $\mathrm{Al}_{n-1} \mathrm{Cu}^{-}$clusters. The open black squares are heat capacities recorded for $\mathrm{Al}_{n}^{+}$clusters (from Refs. 46 and 52). The solid lines are spline fits. The plots are labeled with the total number of atoms in the cluster $(n)$. The dashed lines show heat capacities derived from a modified Debye model (Ref. 53).

Peaks in the heat capacities indicate melting transitions. The size and width of the peaks for $\mathrm{Al}_{n-1} \mathrm{Cu}^{-}$clusters vary over a wide range. The peaks for $n=56$ and 57 are large and narrow, while those for $n=51$ and 52 are small and broad. The center of the peak in the heat capacity is usually taken to be the melting temperature. The area under the peak is the latent heat. For clusters with $n=50-55$ the peak in the heat capacities for $\mathrm{Al}_{n-1} \mathrm{Cu}^{-}$clusters occurs at a lower temperature than for $\mathrm{Al}_{n}^{+}$while for $n=56-62$ (except $n=59$ ) the situation is reversed and the peak for $\mathrm{Al}_{n-1} \mathrm{Cu}^{-}$clusters occurs at a higher temperature than for $\mathrm{Al}_{n}^{+}$. There are also significant differences in the size and width of the peaks. For example, the peak in the heat capacity for $\mathrm{Al}_{56}^{+}$is much broader and smaller than for $\mathrm{Al}_{55} \mathrm{Cu}^{-}$.

In addition to the main peak, there is a small peak in the heat capacity plot for $\mathrm{Al}_{53} \mathrm{Cu}^{-}(n=54)$ which precedes the main melting transition. This feature is reproducible. There is a dip in the heat capacities for some clusters at temperatures below the peak in the heat capacity. For $\mathrm{Al}_{n-1} \mathrm{Cu}^{-}$clusters, a dip occurs for $n=55,56,57$, and 58. The dip also occurs for some $\mathrm{Al}_{n}^{+}$clusters $(n=56,57,58,60,61$, and 62). For $n$ $=56,57$, and 58 the dips for $\mathrm{Al}_{n-1} \mathrm{Cu}^{-}$occur at a significantly lower temperature than for $\mathrm{Al}_{n}^{+}$. The dips for the $\mathrm{Al}_{n}^{+}$clusters have been attributed to freezing into a high energy geometry 
on rapid cooling and then annealing into a lower energy geometry during the longer timescale for transit through the temperature variable extension. ${ }^{52}$

As in our previous studies of the melting of $\mathrm{Al}_{n}^{+}$clusters we fit the peaks in the heat capacities with simple two and three-state models. ${ }^{54}$ Our approach follows that of Poland. ${ }^{19}$ For clusters in the size regime examined here, melting and freezing are thought to occur in the dynamic coexistence regime where the transitions are between fully liquidlike and fully solidlike clusters. This behavior has been observed in computer simulations. ${ }^{55-58}$ In this limit, the liquid and solid are in equilibrium characterized by an equilibrium constant given by

$$
K(T)=\exp \left[-\frac{\Delta H_{\mathrm{MELT}}}{R}\left(\frac{1}{T}-\frac{1}{T_{\mathrm{MELT}}}\right)\right],
$$

where $\Delta H_{\text {MELT }}$ is the latent heat, $T_{\text {MELT }}$ is the melting temperature, and $R$ is the gas constant. The contribution of the latent heats to the heat capacity is

$$
C(T)=\frac{d E_{\mathrm{int}}}{d T}=\frac{\Delta\left(-f_{S}(T) \Delta H_{\mathrm{MELT}}\right)}{\Delta T},
$$

where $f_{S}(T)$ is the fraction of solid present at temperature $T$. Equation (2) describes the peak in the heat capacity that results from the melting transition. This peak is fit to the measured peak using a least squares procedure. Some examples are shown in Fig. 2.

We start by considering the bottom of each plot where the solid lines show the fractions of the solid (light green) and liquid (dark green) that result from the fit. The blue dashed-dotted line shows the contribution to the heat capacity from the latent heat. In order to fit this peak to the experimental results it is necessary to have values for the component of the heat capacity that results from the internal energy of the solid and liquid. For the solid we use the in ternal energy derived from the modified Debye model multiplied by a scale factor $\left(S_{S}\right)$, and for the liquid we use the solid value multiplied by an additional scale factor $\left(S_{L}\right)$ (see Ref. 54 for a full description). Thus, there are four adjustable parameters that are optimized in the least squares fit of the two-state model to the experimental results: $\Delta H_{\mathrm{MELT}}, T_{\mathrm{MELT}}$, $S_{S}$, and $S_{L}$.

Consider the results for $\mathrm{Al}_{52} \mathrm{Cu}^{-}(n=53)$ in the top left panel of Fig. 2. The filled black squares are the experimental values. The open blue circles show the least squares fit calculated with a value for $\Delta T$ in Eq. (2) of $50 \mathrm{~K}$ (the same value as used in the experiments). The solid blue line shows the simulation with a value for $\Delta T$ in Eq. (2) of $5 \mathrm{~K}$. The fact that the calculations with $\Delta T=5$ and $50 \mathrm{~K}$ are similar indicates that the value of $\Delta T$ used in the measurements is small enough that the shape of the peak is faithfully reproduced. It is evident that the two-state model provides a good fit to the experimental results for $\mathrm{Al}_{52} \mathrm{Cu}^{-}$.

The top right panel in Fig. 2 shows results for $\mathrm{Al}_{56} \mathrm{Cu}^{-}$ $(n=57)$. The peak in the heat capacity for this cluster is large and narrow but it is still well fit by the two-state model. In this case the simulation with $\Delta T=5 \mathrm{~K}$ appears sharper than obtained using $\Delta T=50 \mathrm{~K}$. Both $\mathrm{Al}_{52} \mathrm{Cu}^{-}$and $\mathrm{Al}_{56} \mathrm{Cu}^{-}$have

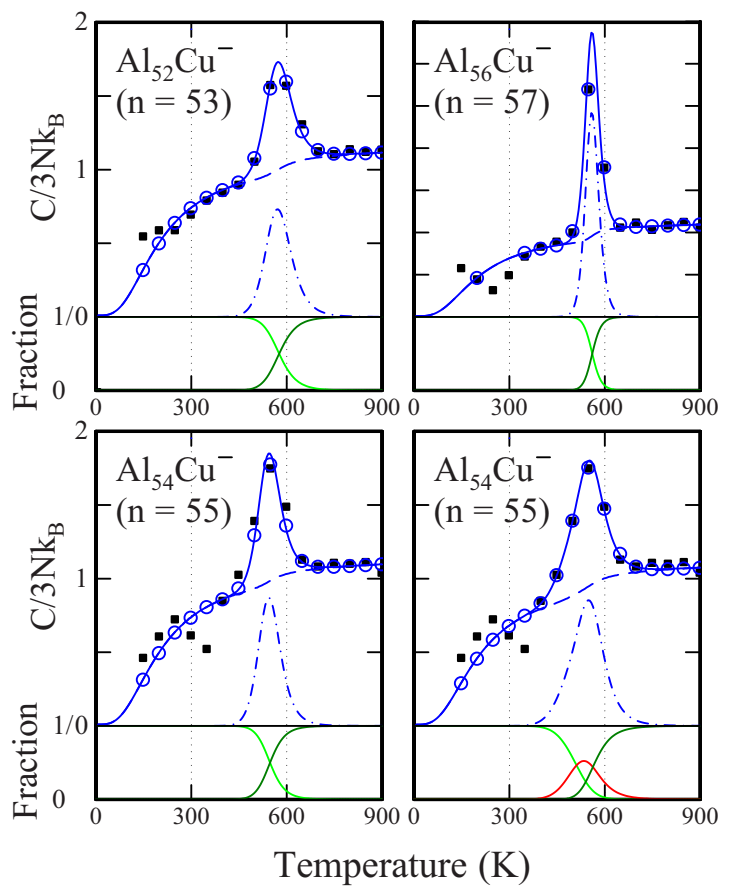

FIG. 2. (Color online) Examples of fits of the two and three-state models (see text) to the experimental results. Fits of the two-state model to the experimental results are shown for $\mathrm{Al}_{52} \mathrm{Cu}^{-}(n=53)$ (top left), $\mathrm{Al}_{56} \mathrm{Cu}^{-}(n$ =57) (top right), and $\mathrm{Al}_{54} \mathrm{Cu}^{-}(n=55)$ (bottom left). Note that the vertical scale for $\mathrm{Al}_{56} \mathrm{Cu}^{-}(n=57)$ is more extended than for the other clusters. The filled black squares are the experimental results, the open blue circles are the fits with $\Delta T$ in Eq. (2) set to $50 \mathrm{~K}$ (as in the experiments). The solid blue line shows the result of the simulation with $\Delta T=5 \mathrm{~K}$. The dashed-dotted lines show the components of the heat capacity due to the latent heat. The solid lines at the bottom of each plot shows the relative abundances of the solidlike (light green) and liquidlike (dark green) clusters as a function of temperature. The fit of the three-state model to the experimental results for $\mathrm{Al}_{54} \mathrm{Cu}^{-}(n=55)$ is shown in the bottom right panel. The solid lines at the bottom of this plot show the relative abundances of the solid (light green), intermediate (red), and liquid (dark green).

similar melting temperatures. The difference between the two clusters is the size of $\Delta \mathrm{H}_{\mathrm{MELT}}: 1.05 \mathrm{eV}$ for $\mathrm{Al}_{52} \mathrm{Cu}^{-}$and $1.94 \mathrm{eV}$ for $\mathrm{Al}_{56} \mathrm{Cu}^{-}$. As the latent heat becomes larger, the peak in the heat capacity becomes narrower.

The peaks for the other $\mathrm{Al}_{n-1} \mathrm{Cu}^{-}(n=49-62)$ clusters studied here can all be fit with the two-state model. For some clusters, the calculated peak is slightly narrower than the measured one. The worst fit is obtained for $\mathrm{Al}_{54} \mathrm{Cu}^{-}(n$ $=55$ ) shown in the lower left panel in Fig. 2. A plausible explanation for this behavior is that melting occurs through a partially melted intermediate (I): $\mathrm{S} \rightleftarrows \mathrm{I} \rightleftarrows \mathrm{L}$. A three-state model can be fit to the experimental results in a manner similar to the two-state model (see Ref. 54 for details). The fit obtained with a three-state model for $\mathrm{Al}_{54} \mathrm{Cu}^{-}(n=55)$ is shown in the lower right panel in Fig. 2. The solid lines at the bottom of this plot show the fractions of the solid (light green), intermediate (red) and liquid (dark green) that result from the fit. Clearly, the small discrepancy in the width of the peak in the heat capacity, that occurred for $\mathrm{Al}_{54} \mathrm{Cu}^{-}(n$ $=55$ ) with the two-state model, has been fixed with the threestate model. With the three-state model there is a melting temperature and latent heat associated with both steps $(\mathrm{S} \rightleftarrows \mathrm{I}$ and $\mathrm{I} \rightleftarrows \mathrm{L})$. For $\mathrm{Al}_{54} \mathrm{Cu}^{-}(n=55)$, the melting temperatures are 541.6 and $559.4 \mathrm{~K}$ compared to $547.4 \mathrm{~K}$ obtained with 


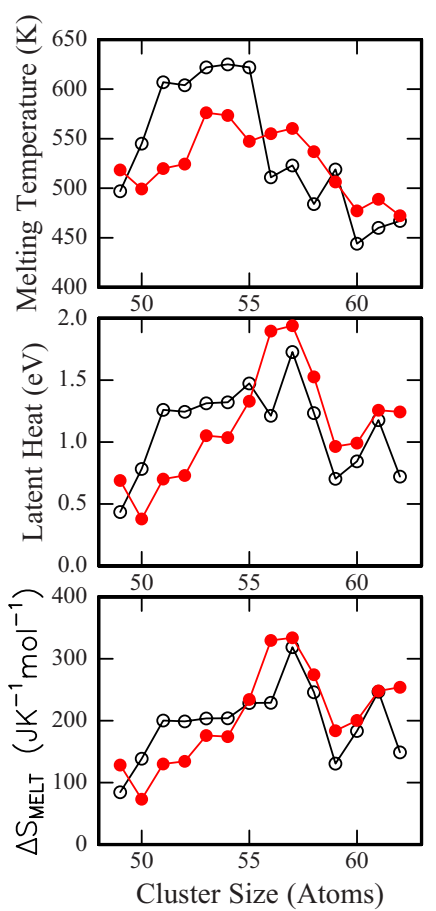

FIG. 3. (Color online) The top panel shows melting temperatures determined from the two-state fits to the experimental results. The filled red circles are the measured values for $\mathrm{Al}_{n-1} \mathrm{Cu}^{-}$clusters. The open black circles are for $\mathrm{Al}_{n}^{+}$clusters (from Refs. 46 and 52). The middle panel shows the latent heats determined from the area of the peak in the heat capacities for $\mathrm{Al}_{n-1} \mathrm{Cu}^{-}$(filled red circles) and $\mathrm{Al}_{n}^{+}$(open black circles). The bottom panel shows the entropy changes for melting $\left(\Delta S_{\mathrm{MELT}}\right)$ (see text) for $\mathrm{Al}_{n-1} \mathrm{Cu}^{-}$ (filled red circles) and $\mathrm{Al}_{n}^{+}$(open black circles).

the two-state fit; and the latent heats are 0.66 and $0.73 \mathrm{eV}$ compared with $1.11 \mathrm{eV}$ obtained with the two-state fit. For the other $\mathrm{Al}_{n-1} \mathrm{Cu}^{-}$clusters $(n=49-62)$ studied here the discrepancy between the measured peak and the two-state fit is smaller than for $\mathrm{Al}_{54} \mathrm{Cu}^{-}(n=55)$. In these cases, the threestate model provides a slightly better fit, but the difference is small and probably not significant.

Substituting the copper atom changes the shape of the heat capacity peaks for some clusters. For example, the heat capacity peaks for $\mathrm{Al}_{51}^{+}$and $\mathrm{Al}_{52}^{+}$have a low temperature shoulder which has been interpreted as evidence for a premelting transition. ${ }^{46}$ The peaks for $\mathrm{Al}_{50} \mathrm{Cu}^{-}$and $\mathrm{Al}_{51} \mathrm{Cu}^{-}$are smaller and broader, and both can be well fit by the two-state model. The peak for $\mathrm{Al}_{56}^{+}$is broad and is not well fit by the two-state model. In contrast, the peak for $\mathrm{Al}_{55} \mathrm{Cu}^{-}$is much sharper and is well fit by the two-state model.

The top panel in Fig. 3 shows melting temperatures determined from the fits with the two-state model. The filled red circles show melting temperatures obtained for $\mathrm{Al}_{n-1} \mathrm{Cu}^{-}$ clusters. The open black circles show values for $\mathrm{Al}_{n}^{+}$clusters. The sharp drop in the melting temperature for $\mathrm{Al}_{n}^{+}$clusters that occurs on going from $n=55$ to 56 is absent for the $\mathrm{Al}_{n-1} \mathrm{Cu}^{-}$clusters.

The middle panel in Fig. 3 shows latent heats determined for $\mathrm{Al}_{n-1} \mathrm{Cu}^{-}$clusters (filled red circles) and $\mathrm{Al}_{n}^{+}$clusters (open black circles). The latent heats come primarily from the fits of the two-state model, but corrected for clusters where the fit is not ideal. The latent heats for the $\mathrm{Al}_{n-1} \mathrm{Cu}^{-}$ clusters range from under $0.5 \mathrm{eV}$ to almost $2 \mathrm{eV}$. For clus- ters with $n=50-55$ the latent heats for $\mathrm{Al}_{n}^{+}$are larger than for $\mathrm{Al}_{n-1} \mathrm{Cu}^{-}$, while for clusters with $n>55$ the $\mathrm{Al}_{n-1} \mathrm{Cu}^{-}$clusters have the larger latent heats.

The entropy changes on melting, shown in the bottom panel of Fig. 3, are obtained from $\Delta S_{\mathrm{MELT}}=\Delta H_{\mathrm{MELT}} / T_{\mathrm{MELT}}$. The filled red circles are for $\mathrm{Al}_{n-1} \mathrm{Cu}^{-}$clusters and the open black circles are for $\mathrm{Al}_{n}^{+}$clusters. The latent heats and the entropies of melting are closely correlated. This explains why the latent heats can vary by more than a factor of four while the melting temperatures for $\mathrm{Al}_{n-1} \mathrm{Cu}^{-}$clusters vary by much less and all lie between 450 and $600 \mathrm{~K}$.

\section{PRELIMINARY DISCUSSION OF EXPERIMENTAL RESULTS}

The $\mathrm{Al}_{n-1} \mathrm{Cu}^{-}$clusters dissociate by sequential loss of neutral aluminum atoms. In the case of $\mathrm{Al}_{48} \mathrm{Cu}^{-}$, for example, we observed the loss of 16 consecutive aluminum atoms as the initial translational energy is raised, and no evidence for the loss of the copper atom. ${ }^{59}$ One might interpret this result as indicating that the copper atom is sequestered away from the surface of the liquid cluster (dissociation occurs from the liquidlike state). However, dissociation here is statistical and hence favors the lowest energy products. Thus, the absence of the copper loss process may simply result because the $\mathrm{Al}_{n-2} \mathrm{Cu}^{-}+\mathrm{Al}$ product is lower in energy than $\mathrm{Al}_{n-1}^{-}+\mathrm{Cu}$. However, we do not rule out the idea that the $\mathrm{Cu}$ atom segregates away from the surface in the liquid cluster. In simulations of $\mathrm{Au}_{54} \mathrm{Cu}$ and $\mathrm{Au}_{43} \mathrm{Cu}_{12}$, Cheng et al. ${ }^{30}$ found that the copper segregated away from the surface of the liquid clusters. It is well known that concentrations at the surfaces of multicomponent liquids often differ from the concentrations in the bulk. Usually the component with the lower surface tension segregates to the surface. ${ }^{60,61}$ In the present case, liquid copper has a higher surface tension than liquid aluminum, ${ }^{62,63}$ and so the copper might be expected to segregate away from the liquid surface.

Bulk aluminum melts at $933 \mathrm{~K}$ and so the melting temperatures of the $\mathrm{Al}_{n}^{+}$clusters shown in Fig. 3 (top panel) are all substantially below the bulk melting point, as expected. ${ }^{23}$ For the $\mathrm{Al}_{n-1} \mathrm{Cu}^{-}$clusters studied here, the atomic composition is $98.0 \%-98.4 \%$ aluminum. For bulk $\mathrm{Al}-\mathrm{Cu}$ alloys with these compositions, melting starts at the eutectic point at $821 \mathrm{~K}$ and finishes below the melting point of the host aluminum metal. ${ }^{64}$ For the $\mathrm{Al}_{n-1} \mathrm{Cu}^{-}$clusters, substituting a copper atom does not always lower the melting temperature (as it does in the bulk). $\mathrm{Al}_{n-1} \mathrm{Cu}^{-}$clusters with $n=56-62$ have melting temperatures that are larger than the corresponding pure aluminum cluster.

Perhaps the most striking difference between the melting behavior of the $\mathrm{Al}_{n}^{+}$and $\mathrm{Al}_{n-1} \mathrm{Cu}^{-}$clusters is the absence, for the $\mathrm{Al}_{n-1} \mathrm{Cu}^{-}$clusters, of the sharp drop in the melting temperature that occurs for $\mathrm{Al}_{n}^{+}$clusters with 56 atoms. This difference propagates to the latent heats: The slight drop for $\mathrm{Al}_{n}^{+}$clusters with 56 atoms is substituted by a large increase for the doped clusters. The significant increase in melting temperature and latent heat observed between $\mathrm{Al}_{49}^{+}$to $\mathrm{Al}_{51}^{+}$is also absent for the copper-alloy counterparts. The drop in the melting temperature for $\mathrm{Al}_{n}^{+}$clusters with 56 atoms was initially attributed to a geometry change ${ }^{65}$ but more recent the- 
oretical results ${ }^{45}$ suggest the structures of $\mathrm{Al}_{55}^{+}$and $\mathrm{Al}_{56}^{+}$at $0 \mathrm{~K}$ are similar. According to the more recent calculations, $\mathrm{Al}_{55}^{+}$shows an enhanced stability because it is an electron shell closing, so the drop in melting temperature for $\mathrm{Al}_{56}^{+}$ might also be induced by the opening of a new electron shell. The electron shell closing for $\mathrm{Al}_{55}^{+}$has been confirmed by photoionization experiments ${ }^{66}$ but, considering the computational difficulties of locating the global minimum energy structures via first-principles calculations, we think theory cannot yet reliably reject the possibility of a structural change.

If the sharp drop in the melting temperatures at $\mathrm{Al}_{56}^{+}$is due to a structural change, then the absence of this drop in the results for the $\mathrm{Al}_{n-1} \mathrm{Cu}^{-}$clusters suggests that the structural change no longer occurs. On the other hand, the latent heats of the substituted and unsubstituted clusters show similar trends for clusters with more than 56 atoms, which suggests structural similarities. Which structure do the $\mathrm{Al}_{n-1} \mathrm{Cu}^{-}$ clusters have? In order to shed some light on this complex problem, we have employed first-principles calculations as detailed in the next section.

\section{COMPUTATIONAL METHODS}

Calculations were performed at the Kohn-Sham density-functional-theory ${ }^{67}$ level to search for the global minimum structures of $\mathrm{Al}_{n-1} \mathrm{Cu}^{-}$anions with $n=49-62$. We employ the SIESTA code, ${ }^{68}$ with exchange and correlation effects treated within the spin-polarized generalized gradient approximation (GGA), ${ }^{69}$ and norm-conserving pseudopotentials to describe the core electrons. ${ }^{70,71}$ The $\mathrm{Al}$ and $\mathrm{Cu}$ atoms contribute 3 and 11 electrons, respectively, to the valence electron density. The copper pseudopotential further includes non-linear core corrections accounting for the core-valence overlap. The basis set employed to expand the cluster wave function contains five basis functions per atom (a double zeta plus polarization or DZP basis in standard notation ${ }^{68}$ ). The spatial extension of the basis functions is determined by an energy shift ${ }^{68}$ of $20 \mathrm{meV}$. The fast-Fourier-transform mesh employed to evaluate some terms in the Hamiltonian is determined by a mesh cutoff ${ }^{68}$ of $100 \mathrm{Ry}$. The quality of the chosen basis set and pseudopotential was tested by performing calculations for the $\mathrm{Al}_{2}$ and $\mathrm{AlCu}$ diatomic molecules as well as for bulk aluminum and copper, with results in good agreement with experiment and previous calculations at the same level of theory.

In a recent work, ${ }^{45}$ we obtained the global minimum energy structures of $\mathrm{Al}_{n}^{+}$cations with $n=34-84$ by using the same theoretical framework. For each cluster size, more than 100 isomers, including icosahedral, decahedral, facecentered-cubic (fcc), and other disordered or mixed structures, were optimized. With this large structural database available, we first tested how the relative stabilities of pure aluminum clusters are modified by a substitutional copper impurity. Specifically, we have considered all the different structural isomers of $\mathrm{Al}_{49}^{+}$to generate initial structures for $\mathrm{Al}_{48} \mathrm{Cu}^{-}$. Each isomer of the pure aluminum clusters leads to several different homotops ${ }^{72}$ for the doped cluster, according to the number of geometrically different substitutional sites.
The set of homotops of a given geometric structure spans an energy interval of width of $0.4-0.5 \mathrm{eV}$. The energy isomer spectrum of the doped cluster is therefore much denser than the pure aluminum reference spectrum. If we now consider only the lowest energy homotop for each structural isomer, the results show that the relative energies of the different isomers are essentially the same in doped and pure clusters. Only when the total energy difference between two $\mathrm{Al}_{49}^{+}$isomers is small (on the order of $0.2 \mathrm{eV}$ ) can substituting copper exchange their energetic ordering.

As noted in the Introduction, calculations suggest that silver and gold icosahedra are stabilized by sequestering a smaller atom at the center. In our previous work, ${ }^{45}$ we found that the $\mathrm{Al}_{55}^{+}$icosahedron lies approximately $3 \mathrm{eV}$ above the ground state structure. We checked the corresponding $\mathrm{Al}_{54} \mathrm{Cu}^{-}$icosahedron (with the copper impurity sequestered at the center), and it lies $2.9 \mathrm{eV}$ above the ground state structure. The icosahedron is thus only marginally stabilized by the copper impurity.

In summary, doping with a single copper impurity is not found to drastically change the structures of aluminum clusters. An important outcome of this observation is that the search for the global minimum energy structures of the doped clusters is simplified. For all other sizes $(n=50-62)$, we have just considered 10-20 low-energy $\mathrm{Al}_{n}^{+}$isomers as candidates to house the copper impurity. We can also reliably conclude that, if the correct structure for $\mathrm{Al}_{n-1} \mathrm{Cu}^{-}$is not found, this is only because the corresponding $\mathrm{Al}_{n}^{+}$global minimum was not located in our previous study. That is, any mistake in the structures of the pure clusters will necessarily transfer to the doped clusters.

\section{THEORETICAL RESULTS}

A representative selection of cluster structures is shown in Fig. 4, and the atomic coordinates for the putative global minima will be made available through the internet. ${ }^{73}$ Within the size range of interest here $(n=49-62)$, the $\mathrm{Al}_{n}^{+}$reference structures are either distorted decahedral fragments (for $n$ $=50-59$ ) or close-packed structures built by stacking planes of atoms according to a fcc sequence (for $n=49,60-62$ ). Within this second structural family, the staking sequence can be locally changed to hcp at the cluster surface, which creates stacking faults in the cluster structure (see Ref. 45 for details). The minimum energy isomer of $\mathrm{Al}_{n-1} \mathrm{Cu}^{-}$has the same structure as the corresponding $\mathrm{Al}_{n}^{+}$global energy minimum with few exceptions: for $n=54$ the doped structure pertains to the same distorted decahedral family as the parent pure cluster, but differs slightly from it by the reallocation of a few surface atoms; for $n=56-59$, distorted decahedra and close-packed structures are degenerate in energy (the small energy differences between these isomers, smaller than $1 \mathrm{meV} /$ atom, are clearly beyond the numerical accuracy expected from DFT calculations). Therefore, the critical size for the transition from distorted decahedral to close-packed structures, which occurs at $n=60$ in the pure clusters, is lowered to $n=56$ in the doped clusters within our numerical accuracy.

Previous $a b$ initio calculations on smaller aluminum 

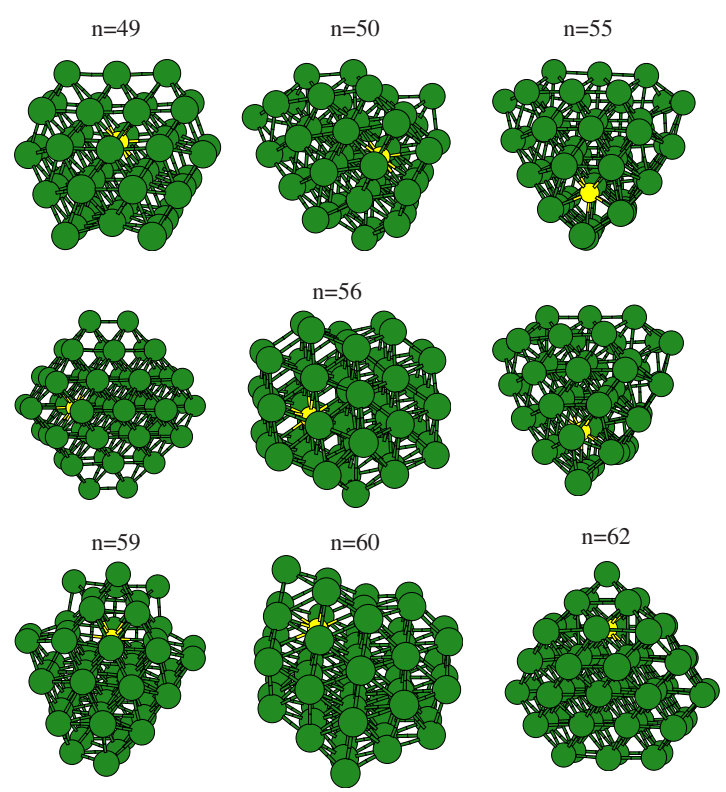

FIG. 4. (Color online) A representative selection of the structures adopted by $\mathrm{Al}_{n-1} \mathrm{Cu}^{-}$cluster anions. The yellow (light) sphere represents the $\mathrm{Cu}$ impurity. The total number of atoms $(n)$ is given above each structure. For $n=56$ we show three nearly degenerate isomers. All the clusters with $n$ $=50-55$ share the same structural motif, so we show only two structures explicitly. The same happens within the size ranges $n=57-59$ and $n$ $=60-61$.

clusters doped with copper ${ }^{74-78}$ do not fully agree on the preferred substitution site for the copper atom. Our results show that copper prefers to occupy interior positions in host aluminum clusters with 49-62 atoms, although the energetic cost for surface segregation of copper is not very large: The most stable homotop with copper at the cluster surface is roughly $0.2 \mathrm{eV}$ above the correct ground state. There are some exceptions to this rule: for $n=61$, for example, there is one homotop with the copper atom at the surface which is essentially degenerate with the ground state. For clusters with $n=50-55$, the copper impurity does not occupy the most internal positions, but intermediate ones. These are sites which may be considered as internal in terms of the coordination number of copper but which lack a capping aluminum atom, so that the impurity is partially exposed to the cluster surface (this is most clearly seen for $n=55$ in Fig. 4).

From all the possible interior sites available for substitution, the copper impurity further shows a strong preference for those providing $12 \mathrm{Al}-\mathrm{Cu}$ bonds in a local fcc atomic environment. All the host aluminum clusters considered here possess at least one such substitution site, but in many cases (especially within the distorted decahedral family) the local fcc atomic environment is considerably distorted, and the 12 $\mathrm{Al}-\mathrm{Al}$ bond lengths around the substituted aluminum show a large dispersion. Host clusters with $n=49,56$ (the left isomer in Fig. 4) and 62, however, offer an almost perfect local fcc environment around the substitution site. As we will see below, these undistorted sites are able to accommodate the relaxation around the smaller $\mathrm{Cu}$ impurity much more efficiently than the distorted fcc environments. For $n=54$ and $n=55$, the local fcc environment is so distorted that the copper impurity prefers a different substitution site closer to the cluster surface (see Fig. 4), where it is coordinated to only

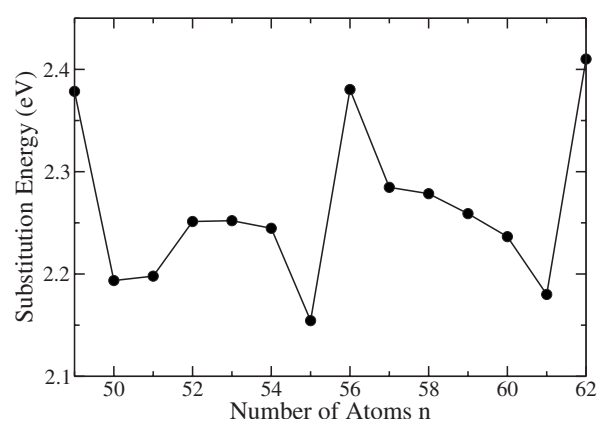

FIG. 5. Energy released after substitutional doping with copper, as a function of the cluster size $n$ (total number of atoms). The doping process, as described in the text, does not change the total number of valence electrons in the cluster (the $10 \mathrm{~d}$-electrons of the copper atom are not considered as valence electrons in this discussion).

10 aluminum atoms instead of 12 . Clusters with $n=54$ and 55 can thus be considered as exceptional cases with respect to the average structural trend.

According to the identified structural trend, pure aluminum clusters with a close-packed structure are expected to provide a more optimal atomic environment for the copper impurity, as compared to the distorted decahedral clusters. In fact, we observe the energy difference between distorted decahedral and close-packed structures to decrease by about $0.2 \mathrm{eV}$ upon substituting with a copper atom. For $n$ $=50-55$ atoms, the distorted decahedral structures are significantly more stable than the close-packed structures, so the doped clusters still adopt the distorted decahedral structure, even if the local atomic environment for copper is not optimal. For $n=56-59$, both structural families are sufficiently close in energy for the pure clusters, so that substituting with copper can induce a structural change, as seen in Fig. 4.

As we are mainly interested in a comparison between pure and substituted aluminum clusters, we show in Fig. 5 the energy released in the exothermic substitution reaction $\mathrm{Al}_{n}^{+}+\mathrm{Cu}^{-} \rightarrow \mathrm{Al}_{n-1} \mathrm{Cu}^{-}+\mathrm{Al}^{+}$. This "substitution energy" shows marked maxima for sizes $n=49,56$, and 62 , precisely those offering an undistorted fcc-like atomic environment to the copper impurity. A comparison with Fig. 3 reveals that those three sizes have the largest latent heat increase upon doping. In particular, the cluster with $n=56$ atoms is stabilized by doping with a copper atom much more than the cluster with $n=55$ atoms, which also correlates with the qualitative change in the size evolution of the latent heats on passing from 55 to 56 atoms. For those clusters with more than 56 atoms, $n=61$ has the smallest latent heat increase upon doping with copper (see Fig. 3), which correlates with a local minimum in the substitution energy. Finally, the energy gained in the doping process is on average larger for $n=49$ and $n \geqslant 56$ than for the $n=50-55$ size range, which also qualitatively correlates with the doping-induced changes in the latent heats. On the quantitative level, however, the variations of the substitution energy with cluster size are significantly smaller than the corresponding latent heat changes.

\section{DISCUSSION}

Introduction of a substitutional impurity is expected to modify the free energies of both solidlike and liquidlike 
phases. If the free energy difference between liquid and solid phases decreases upon doping, the doped cluster will show a depressed melting point and latent heat. In the opposite case, the solid phase of the doped cluster will have enhanced thermal stability and therefore a higher melting point than the pure cluster. Let us make the reasonable assumption that the copper-induced free energy change evolves smoothly with size for the liquid clusters. Then, when the doped cluster has, for example, a larger latent heat than the pure cluster, it must be because the solid substitution energy evaluated in the previous section is especially large. This provides a rationale for the good qualitative correlation observed between the theoretical solid substitution energies and the measured latent heat changes.

There are several different melting scenarios possible in bimetallic nanoalloys. When the two metals segregate in the solid phase but mix in the liquid phase, melting points are systematically depressed. This is observed, for example, in the melting transition of alkali nanoalloys of several compositions. ${ }^{32}$ The main reason is the mixing entropy, which strongly favors the liquid phase in this case. This is also the reason why the melting points of bulk alloys in the dilute limit are systematically depressed, so it is a wellknown effect. When the two metals mix both in solid and liquid phases, the behavior is in general unpredictable: The melting points may be either depressed or enhanced, depending on the specific system. This is also true both for nanoalloys and bulk alloys. Finally, when the two elements are immiscible both in solid and liquid phases, the bulk melting point of the majority phase is not modified at all by the presence of a small concentration of the minority phase (the two systems behave just independently). In small clusters, however, immiscible impurities are expected to systematically depress the melting temperatures of the host cluster. ${ }^{79}$ The reason is the energetic cost of the interface separating the two components, which can be neglected in the bulk limit but not at the nanoscale. For sufficiently large impurities of general shape, the liquid phase is expected to better accommodate the interfacial region (due to its larger structural freedom), while the more rigid solid phase has to sustain a large interfacial tension. The stress stored in the solid phase is efficiently released upon melting, which promotes the liquid phase and depresses the melting point. ${ }^{79}$

The single atomic impurity considered here represents a special case because it has a perfectly spherical shape (i.e., it is not a molecular impurity). In contrast to bulk systems, pure metallic clusters may on their own sustain a significant degree of bond strain. A single atomic substitutional impurity may put the host lattice under additional stress, but it also may help to ameliorate the existing strain in the host metallic cluster. A specific example of this last situation, which tends to enhance the melting points of the doped clusters, has been shown by Mottet et al.: ${ }^{33}$ Introducing a copper impurity at the center position of an $\mathrm{Ag}_{55}$ icosahedron significantly relaxes the strain of the host cluster because a copper atom is smaller than a silver atom. Note that in this special example the relaxation of the host lattice around the impurity is ho-

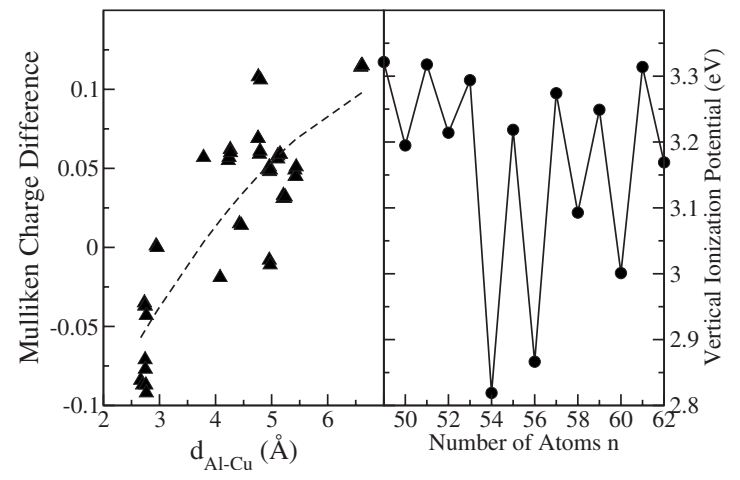

FIG. 6. Left panel: the difference between the aluminum Mulliken charges in doped and pure clusters is plotted as a function of the distance to the $\mathrm{Cu}$ impurity. The dashed line is a simple polynomial fit intended as a guide to the eye. Right panel: vertical ionization potential (VIP) of $\mathrm{Al}_{n-1} \mathrm{Cu}^{-}$, plotted as a function of the total number of atoms $n$. The VIP is the energy difference between the cluster anion and the neutral cluster, with both in the optimum geometry of the anion.

mogeneous, that is, involves the coherent contraction of the outer silver atomic shells without unevenly redistributing the remaining bond strain.

We find a similar physical interpretation for the observed melting peculiarities in $\mathrm{Al}_{n-1} \mathrm{Cu}^{-}$cluster anions. When the host cluster contains an interior site with a perfect fcc-like local environment, the local contraction of the host cluster around the smaller impurity is coherent: All the $\mathrm{Al}-\mathrm{Cu}$ bonds are contracted by similar amounts and the optimal distribution of strain in the host lattice is respected. When the substitution site has a significantly distorted fcc-like atomic environment, structural optimization of the doped cluster tends to make the twelve $\mathrm{Al}-\mathrm{Cu}$ distances as similar as possible and thus significantly perturbs the optimal distribution of strain in the host lattice. For clusters with $n=54$ and 55 atoms, this is so unfavorable for the solid cluster that the copper impurity prefers a different substitution site with only ten $\mathrm{Al}-\mathrm{Cu}$ bonds.

Our interpretation for the changes in melting behavior induced by a substitutional copper impurity is therefore purely structural. However, as $\mathrm{Cu}$ and $\mathrm{Al}$ have different valence states and also different electronegativities, one might expect some contribution to the observed changes coming from electronic effects. So just to complete our view of the doping process, we have analyzed the electronic changes induced by doping. A Mulliken population analysis reveals a negligible charge transfer between $\mathrm{Cu}$ and $\mathrm{Al}$ atoms. This agrees with the results of Zope and Baruah, ${ }^{74}$ who also observed a negligible amount of charge transfer when the copper impurity substitutes an interior aluminum atom. Now, if $\mathrm{Al}_{n}^{+}$and $\mathrm{Al}_{n-1} \mathrm{Cu}^{-}$clusters have the same total number of electrons and the copper impurity is in its monovalent state, the doped clusters must have an excess of electron charge (integrating to two electrons) distributed in the cluster regions far from the impurity. This is shown in Fig. 6 (left panel), where we plot the difference between the aluminum Mulliken charges of the doped and pure 49-atom clusters, as a function of the distance from the copper impurity. The first coordination shell of aluminum atoms around the copper impurity shows a deficit of electron charge, while the more 
distant atoms show an accumulation of electron charge. As the impurity occupies an internal position, the electron excess is mostly accommodated at the cluster surface. This is certainly a small difference between the doped and pure clusters, but one which is systematic as it is essentially the same for all sizes. Apart from this, the copper impurity does not significantly perturb the electronic structure of the host cluster: The vertical ionization potential (VIP) of the doped cluster anions (the energy difference between the cluster anion and the neutral cluster in the optimum geometry of the anion) shown in the right panel of Fig. 6, identifies electronic shell closings for $n=53$ and 55 atoms, in perfect coincidence with the electron shell closings observed for the pure aluminum cations. ${ }^{45,66}$ The different melting behavior of pure and doped clusters is thus not due to different electron shell structures.

The agreement between theory and experiment is encouraging, and possibly as good as might be expected from purely static calculations. As discussed above, theory finds an explanation for the qualitative trends observed in the melting experiments. In the future, explicit molecular dynamics simulations of the melting process might offer a more definitive and complete explanation of the experimental observations, possibly also at the quantitative level.

\section{CONCLUSIONS}

Heat capacities measured for $\mathrm{Al}_{n-1} \mathrm{Cu}^{-}$clusters show peaks that can be attributed to melting transitions. The melting temperatures and latent heats for the $\mathrm{Al}_{n-1} \mathrm{Cu}^{-}$clusters show significant differences from those for isoelectronic $\mathrm{Al}_{n}^{+}$ clusters, confirming theoretical predictions that the melting behavior of metal clusters can be significantly modified by substituting a single metal atom. The sharp drop in the melting temperature that occurs between $n=55$ and 56 for pure aluminum clusters does not occur for the $\mathrm{Al}_{n-1} \mathrm{Cu}^{-}$analogs.

First-principles density-functional calculations reveal that the structures of $\mathrm{Al}_{n}^{+}$clusters are not significantly modified upon doping. The electron shell structure is also the same in $\mathrm{Al}_{n}^{+}$and $\mathrm{Al}_{n-1} \mathrm{Cu}^{-}$clusters, which contain the same total number of electrons. The copper impurity substitutes an internal aluminum atom, preferably one with a local facecentered-cubic atomic environment. Despite the similarities between pure and doped clusters, the detailed atomic relaxation of the host aluminum cluster around the impurity is size dependent, which makes the substitution reaction significantly more exothermic for specific cluster sizes. The main physical factors controlling the strong size dependence of the substitution energy have been identified and discussed. The size dependence of the substitution energy is in qualitative agreement with the modifications in the latent heats of melting induced by the copper impurity. However, several other experimental observations about the melting transition can not be interpreted with purely static (zero Kelvin) calculations.

\section{ACKNOWLEDGMENTS}

We gratefully acknowledge the support of the National Science Foundation, the Spanish MEC, and the European
Regional Development Fund (Project Nos. MAT2005-03415 and VA068A06).

${ }^{1}$ R. Li, J. H. G. Owen, S. Kusano, and K. Miki, Appl. Phys. Lett. 89, 073116 (2006).

${ }^{2}$ Y. J. Lee, E.-K. Lee, S. Kim, and R. M. Nieminen, Phys. Rev. Lett. 86, 999 (2001).

${ }^{3}$ G. A. Breaux, D. A. Hillman, C. M. Neal, R. C. Benirschke, and M. F. Jarrold, J. Am. Chem. Soc. 126, 8628 (2004).

${ }^{4}$ A. Aguado and J. M. López, Phys. Rev. Lett. 94, 233401 (2005).

${ }^{5}$ H. Haberland, T. Hippler, J. Donges, O. Kostko, M. Schmidt, and B. von Issendorff, Phys. Rev. Lett. 94, 035701 (2005).

${ }^{6}$ K. Koga, T. Ikeshoji, and K. Sugawara, Phys. Rev. Lett. 92, 115507 (2004).

${ }^{7}$ F. Ding, A. Rosén, and K. Bolton, Phys. Rev. B 70, 075416 (2004).

${ }^{8}$ F. Chuang, C. Z. Wang, S. Öğüt, J. R. Chelikowsky, and K. M. Ho, Phys. Rev. B 69, 165408 (2004).

${ }^{9}$ D. Schebarchov and S. C. Hendy, Phys. Rev. Lett. 95, 116101 (2005).

${ }^{10}$ A. Aguado and J. M. López, Phys. Rev. B 74, 115403 (2006).

${ }^{11}$ D. M. Tomecka, B. Partoens, and F. M. Peeters, Phys. Rev. E 71, 062401 (2005).

${ }^{12}$ E. G. Noya, J. P. K. Doye, D. J. Wales, and A. Aguado, Eur. Phys. J. D 43, 57 (2007).

${ }^{13}$ M. Lee, S. Gowtham, H. He, K.-C. Lau, L. Pan, and D. G. Kanhere, Phys. Rev. B 74, 245412 (2006).

${ }^{14}$ M. Lee and D. G. Kanhere, Phys. Rev. B 75, 125427 (2007).

${ }^{15}$ A. N. Andriotis, Z. G. Fthenakis, and M. Menon, Phys. Rev. B 75, 073413 (2007).

${ }^{16}$ A. V. Zhukov, A. S. Kraynyukova, and J. Cao, Phys. Lett. A 364, 329 (2007).

${ }^{17}$ M. Eryrüek and M. H. Güven, Physica A 377, 514 (2007).

${ }^{18}$ S. Luo, L. Zheng, A. Strachan, and D. C. Swift, J. Chem. Phys. 126, 034505 (2007).

${ }^{19}$ D. Poland, J. Chem. Phys. 126, 054507 (2007).

${ }^{20}$ Y. Teng, X. Zeng, H. Zhang, and D. Shun, J. Phys. Chem. B 111, 2309 (2007).

${ }^{21}$ P. C. R. Rodrigues and F. M. S. Silva Fernandes, Eur. Phys. J. D 41, 113 (2007).

${ }^{22}$ H. Duan, F. Ding, A. Rosén, A. R. Harutyunyan, S. Curtarolo, and K. Bolton, Chem. Phys. 333, 57 (2007).

${ }^{23}$ P. Pawlow, Z. Phys. Chem. (Leipzig) 65, 1 (1909).

${ }^{24}$ L. S. Lai, J. Y. Guo, V. Petrova, G. Ramanath, and L. H. Allen, Phys. Rev. Lett. 77, 99 (1996)

${ }^{25}$ M. Schmidt, R. Kusche, T. Hippler, J. Donges, W. Kronmüller, B. von Issendorff, and H. Haberland, Phys. Rev. Lett. 86, 1191 (2001).

${ }^{26}$ M. Schmidt, T. Hippler, J. Donges, W. Kronmüller, B. von Issendorff, and H. Haberland, Phys. Rev. Lett. 87, 203402 (2001).

${ }^{27}$ M. Schmidt, J. Donges, T. Hippler, and H. Haberland, Phys. Rev. Lett. 90, 103401 (2003)

${ }^{28}$ A. A. Shvartsburg and M. F. Jarrold, Phys. Rev. Lett. 85, 2530 (2000).

${ }^{29}$ G. A. Breaux, R. C. Benirschke, T. Sugai, B. S. Kinnear, and M. F. Jarrold, Phys. Rev. Lett. 91, 215508 (2003).

${ }^{30}$ D. Cheng, S. Huang, and W. Wang, Phys. Rev. B 74, 064117 (2006).

${ }^{31}$ F. Chen, B. C. Curley, G. Rossi, and R. L. Johnston, J. Phys. Chem. C 111, 9157 (2007).

${ }^{32}$ A. Aguado, L. E. González, and J. M. López, J. Phys. Chem. B 108, 11722 (2004); A. Aguado and J. M. López, Phys. Rev. B 71, 075415 (2005); J. Chem. Theory Comput. 1, 299 (2005); Phys. Rev. B 72, 205420 (2005); A. Aguado, J. M. López, and S. Núñez, Comput. Mater. Sci. 35, 174 (2006)

${ }^{33}$ C. Mottet, G. Rossi, F. Baletto, and R. Ferrando, Phys. Rev. Lett. 95, 035501 (2005).

${ }^{34}$ K. Michaelian and I. L. Garzón, Eur. Phys. J. D 34, 183 (2005).

${ }^{35}$ T. T. Järvi, A. Kuronen, K. Meinander, K. Nordlund, and K. Albe, Phys. Rev. B 75, 115422 (2007).

${ }^{36}$ M. S. Lee, S. Gowtham, H. He, K.-C. Lau, L. Pan, and D. G. Kanhere, Phys. Rev. B 74, 245412 (2006).

${ }^{37}$ I. Kaban, I. Kaban, W. Hoyer, A. Il'inskii, A. Shpak, and P. Jóvári, J. Non-Cryst. Solids 353, 1808 (2007).

${ }^{38}$ J. T. Okada, M. Inui, D. Ishikawa, A. Q. R. Baron, K. Matsuda, S. Tsutsui, Y. Watanabe, S. Nanao, and T. Ishikawa, J. Phys.: Condens. Matter 18, L613 (2006).

${ }^{39}$ X. Lv, X. Bian, T. Mao, Z. Li, J. Guo, and Y. Zhao, Physica B 392, 34 (2007). 
${ }^{40}$ T. Yen, S. K. Lai, N. Jakse, and J. L. Bretonnet, Phys. Rev. B 75, 165420 (2007).

${ }^{41}$ K. Manninen, A. Rytkönen, and M. Manninen, Eur. Phys. J. D 29, 39 (2004).

${ }^{42}$ R. Ferrando, J. Jellinek, and R. L. Johnston, Chem. Rev. (Washington, D.C.) 108,845 (2008).

${ }^{43}$ J. Jellinek, Faraday Discuss. 138, 11 (2008).

${ }^{44}$ C. M. Neal, A. K. Starace, and M. F. Jarrold, J. Phys. Chem. A 111, 8056 (2007)

${ }^{45}$ A. K. Starace, C. M. Neal, B. Cao, M. F. Jarrold, A. Aguado, and J. M. López, "Correlation between the latent heats and cohesive energies of metal clusters and change," J. Chem. Phys. (in press).

${ }^{46}$ G. A. Breaux, C. M. Neal, B. Cao, and M. F. Jarrold, Phys. Rev. Lett. 94, 173401 (2005).

${ }^{47}$ C. M. Neal, A. K. Starace, and M. F. Jarrold, J. Am. Soc. Mass Spectrom. 18, 74 (2007)

${ }^{48}$ C. M. Neal, G. A. Breaux, B. Cao, A. K. Starace, and M. F. Jarrold, Rev. Sci. Instrum. 78, 075108 (2007).

${ }^{49}$ M. F. Jarrold and E. C. Honea, J. Am. Chem. Soc. 114, 459 (1992).

${ }^{50}$ M. F. Jarrold, J. Phys. Chem. 99, 11 (1995).

${ }^{51}$ In the model used to estimate the fraction of the ions translational energy that is converted into internal energy, the energy transfer depends on the mass of the atoms in the cluster. According to the model, the energy transferred by a collision with a copper atom is around $54 \%$ less than the energy transferred in a collision with an aluminum atom. We assume that the probability of collision with a copper atom is proportional to the fraction of copper atoms in the cluster. Thus an error would result if the copper atom is not distributed statistically (i.e., if the copper atom segregated away from the surface layer or into the surface layer). However, because the number of impurity atoms is small compared to the number of host atoms in the clusters studied here, the potential error is small (around 1\%)

${ }^{52}$ M. F. Jarrold, B. Cao, A. K. Starace, C. M. Neal, and O. H. Judd, J. Chem. Phys. 129, 014503 (2008)

${ }^{53}$ J. Bohr, Int. J. Quantum Chem. 84, 249 (2001).

${ }^{54}$ C. M. Neal, A. K. Starace, and M. F. Jarrold, Phys. Rev. B 76, 054113 (2007).

${ }^{55}$ R. S. Berry, J. Jellinek, and G. Natanson, Phys. Rev. A 30, 919 (1984).

${ }^{56}$ T. L. Beck, J. Jellinek, and R. S. Berry, J. Chem. Phys. 87, 545 (1987).

${ }^{57}$ J. P. Rose and R. S. Berry, J. Chem. Phys. 98, 3246 (1993).

${ }^{58}$ B. Vekhter and R. S. Berry, J. Chem. Phys. 106, 6456 (1997).
${ }^{59}$ If loss of the copper atom occurred with the same probability as loss of an aluminum atom, around $40 \%$ of the starting $\mathrm{Al}_{48} \mathrm{Cu}^{-}$clusters should have lost the copper atom after losing 16 atoms sequentially.

${ }^{60}$ G. Raina, C. U. Kulkarni, and C. R. Rao, J. Phys. Chem. A 105, 10204 (2001)

${ }^{61}$ E. Dimasi, H. Tostman, O. Shpyrkp, P. Huber, B. Ocko, P. Pershan, M. Deutsch, and L. Berman, Phys. Rev. Lett. 86, 1538 (2001).

${ }^{62}$ V. Sarou-Kanian, F. Millot, and J. C. Rifflet, Int. J. Thermophys. 24, 277 (2003).

${ }^{63}$ T. Matsumoto, H. Fujii, T. Ueda, M. Kamai, and K. Nogi, Meas. Sci. Technol. 16, 432 (2005).

${ }^{64}$ R. A. Flinn and P. K. Trojan, Engineering Materials and Their Applications, 4th ed. (Houghton Mifflin, Boston, 1990).

${ }^{65}$ A. Aguado and J. M. López, J. Phys. Chem. B 110, 14020 (2006).

${ }^{66}$ K. E. Schriver, J. L. Persson, E. C. Honea, and R. L. Whetten, Phys. Rev. Lett. 64, 2539 (1990).

${ }^{67}$ W. Kohn and L. J. Sham, Phys. Rev. 140, 1133 (1965).

${ }^{68}$ J. M. Soler, E. Artacho, J. D. Gale, A. García, J. Junquera, P. Ordejón, and D. Sánchez-Portal, J. Phys.: Condens. Matter 14, 2745 (2002).

${ }^{69}$ J. P. Perdew, K. Burke, and M. Ernzerhof, Phys. Rev. Lett. 77, 3865 (1996); 78, 1396 (1997).

${ }^{70}$ D. R. Hamann, M. Schlüter, and C. Chiang, Phys. Rev. Lett. 43, 1494 (1979).

${ }^{71}$ L. Kleinman and D. M. Bylander, Phys. Rev. Lett. 48, 1425 (1982).

${ }^{72} \mathrm{An}$ isomer with the the same geometric structure but with the impurity atom occupying a different site. See, E. B. Krissinel and J. Jellinek, Int. J. Quantum Chem. 62, 185 (1997).

${ }^{73}$ Web page of the "Nanometric Properties of Matter" Research Group: http://metodos.fam.cie.uva.es/ GIR

${ }^{74}$ R. R. Zope and T. Baruah, Phys. Rev. A 64, 053202 (2001).

${ }^{75}$ S. N. Khanna, C. Ashman, B. K. Rao, and P. Jena, J. Chem. Phys. 114, $9792(2001)$

${ }^{76}$ P. Yang, J.-H. Ge, and Z.-Y. Jiang, J. Mol. Struct.: THEOCHEM 755, 75 (2005).

${ }^{77}$ C. Lacaze-Dufaure, C. Blanc, G. Mankowski, and C. Mijoule, Surf. Sci. 601, 1544 (2007).

${ }^{78}$ R. Pal, L. Cui, S. Bulusu, H. Zhai, L. Wang, and X. C. Zeng, J. Chem. Phys. 128, 024305 (2008).

${ }^{79}$ C. Hock, S. Strassburg, H. Haberland, B. V. Issendorff, A. Aguado, and M. Schmidt, Phys. Rev. Lett. 101, 023401 (2008). 